\title{
Beyond COVID-19: respiratory infection and cardiovascular events
}

\author{
Joseph J Lee* \\ Constantinos Koshiaris \\ F D Richard Hobbs \\ James P Sheppard \\ All authors of Nuffield Department of Primary Care Health Sciences, University of Oxford \\ *Corresponding author, joseph.lee@phc.ox.ac.uk
}

When someone has an infection, their risk of having a heart attack or stroke rises abruptly, before returning to pre-infection levels over the next few weeks.[1] The increase in risk is particularly high for respiratory infections like influenza and pneumonia. There are biologically plausible mechanisms by which infections could cause cardiovascular disease (CVD). Respiratory infections could trigger CVD by functional changes, like tachycardia, blood pressure instability, and hypercoagulable states. Increased cardiac demand can be enough to cause myocardial ischaemia downstream of stenosed vessels, even if the obstruction is stable.[2] Infection might worsen or trigger existing cardiovascular disease. Atheroma is thought to result from inappropriate inflammation. Infection could accelerate this inflammatory process, and destabilise plaques, much as chronic inflammatory conditions worsen coronary disease. Infections are also triggers for arrhythmias, which can in turn cause myocardial infarction and stroke. But even if the association between infections and CVD is only a marker of risk and not causative, it could still be useful clinically. There are precedents for short-term interventions: for example temporary dual antiplatelet therapy after TIAs. The benefit of prevention is proportional to the risk of CVD events, so treatments can be beneficial during periods of higher risk, when the balance of risks to benefits favours intervention.

A typical question GPs ask is: "Why has this person got this disease at this time?" Often we focus on the diagnosis and cause, but forget the temporal element, or at least don't take it into account in our practice. At one time, even death was assumed to strike people at random. During the plague, London haberdasher John Graunt started tallying up parish bills of mortality and found the very young and the very old were more likely to die than those lucky enough to be middle aged.[3] So much in our practice flows from this simple fact. That old people die seems obvious now; it is so ingrained as to be hard to imagine not knowing. But how much more sophisticated is our understanding of other seemingly random events? Tallying the deaths from our $21^{\text {st }}$ century plague shows COVID-19 conforms to the pattern of older people being more likely to die. It also highlights the risks of combining infections with cardiovascular disease, with over 31,000 cardiovascular deaths attributed to COVID-19 in 2020.[4] So, can we move on from treating them like they are unrelated? This isn't a small problem: epidemiology from before the COVID-19 pandemic estimates some 10,000 CVD deaths per year may be related to infections.[5] Therefore, that few weeks of increased risk could represent a huge burden of coordinated disease. 
The race is not to the swift, nor the battle to the strong, nor bread to the wise, nor yet favour to men of skill, but time and chance happen to them all.[6]. Does this describe our approach to CVD events in primary care? Not quite; we are not in the pre-Graunt era. We accept patients have different levels of risk attributable to their risk factors. We treat people accordingly - if their ten year CVD risk meets a threshold, we offer statins: this drug is cost effective and safe in the long term, go forth and take them forever. We know this approach saves lives, it is good and necessary, but can we do more? COVID-19 demonstrated the seasonal nature of infections, and the power of epidemiology. This isn't new information; Graunt noticed diseases have seasonal patterns in the seventeenth century.[3] And it's not just infections; those of us working in the NHS refer to seasonal deterioration in chronic diseases as 'winter pressures'.[5] Primary care adapted in response to epidemiology in the last eighteen months, so maybe we can continue to adapt to data on seasonal infections. Infection control drove most of the recent changes in practice. We could continue this focus after the pandemic, doing our best to protect patients with chronic diseases from infections. Remote consulting, separate pathways for infectious patients, and vaccination have a lot to offer. To offer the safest care we have to think about the risks from infections, which includes CVD.

CVD isn't entirely random;; most winters there is a spike in CVD and respiratory infections.[5] We have known for years that respiratory infections are followed by a few weeks of higher CVD risk.[1,7] This association was identified in general practice data, but we don't use it in general practice; we don't mention it to people with high ten-year CVD risk, nor when treating patients for respiratory infections. The only concession is offering people influenza and pneumococcal vaccines for secondary CVD prevention. And how often do we represent this to our patients as preventing bad infections, rather than preventing CVD? Even the official NHS website suggests to patients: "Flu can be very serious if you have coronary heart disease".[8] Shouldn't we mention that influenza vaccines reduce secondary CVD events, and by an impressive margin?[9] The effect of 'flu vaccines is greater than is seen for other secondary prevention measures.[10,11] Perhaps the COVIDrelated expansion of influenza vaccine coverage will help with primary CVD prevention too. We should certainly tally up the primary care data to find out.

We should also think about how to perform short-term CVD risk assessment and intervention when people are unwell with respiratory infections. One of the lessons of COVID-19 is the need for accurate risk prediction to guide vaccination and shielding.[12]. Patients want to know their CVD risk and how to manage it [13] but at the moment we don't know if the risk following infections is simply proportional to ten-year CVD risk, or if we need different tools that take into account risk factors for frequent or severe infections. If we can recognise and predict the risk, we could use infection as a prompt to personalise CVD risk assessments, and perhaps intervene.

Short-term risk assessments are beginning to be brought to bear on CVD; we are starting to intervene at high-risk moments. TIAs mark a peak in stroke risk that lasts for a few weeks. Intervening with short-term dual antiplatelet therapy at this crucial time reduces strokes.[14] And similarly, short courses of dual antiplatelets are used for acute coronary syndrome. Because the treatment is short, the risk of bleeding is likely to be low. This is in contrast to long term aspirin for primary CVD prevention - which does reduce events, but the risk of bleeding outweighs the benefit.[11] Could we apply similar thinking to the high-risk period 
following the onset of infections, identify people at risk of infection-related CVD, and intervene with a short course of antiplatelets?

Risk stratification is key - we know aspirin causes bleeds and reduces CVD by about the same amount in primary prevention. [11] The RECOVERY trial also demonstrated this in patients hospitalised with COVID-19.[15] Aspirin reduced thromboembolic events and increased bleeds, but had no effect on all-cause mortality. Seasonal and infection-related CVD predates the pandemic, and we should explore predicting and preventing CVD related to other respiratory infections as well. We already know infections are associated with CVD events, so let's make use of this fact. As we tally up the bills of mortality from COVID-19, let's think about how infections can trigger us to prevent CVD, rather than allowing infections to trigger CVD events.

Funding:

JJL is funded by the National Institute for Health Research (NIHR). The views expressed are those of the authors and not necessarily those of the NIHR or the Department of Health and Social Care. JS receives funding from the Wellcome Trust/Royal Society via a Sir Henry Dale Fellowship (ref: 211182/Z/18/Z). He also receives funding from the National Institute for Health Research (NIHR) School for Primary Care Research, NIHR Oxford Biomedical Research Centre, British Heart Foundation and Stroke Association. For the purpose of Open Access, the author has applied a CC BY public copyright licence to any Author Accepted Manuscript version arising from this submission. 
1 Smeeth L, Thomas SL, Hall AJ, et al. Risk of Myocardial Infarction and Stroke after Acute Infection or Vaccination. N Engl J Med 2004;351:2611-8. doi:10.1056/NEJMoa041747

2 Thygesen K, Alpert JS, Jaffe AS, et al. Fourth Universal Definition of Myocardial Infarction (2018). J Am Coll Cardiol 2018;72:2231-64. doi:10.1016/j.jacc.2018.08.1038

3 Graunt J. Natural and Political Observations Made Upon the Bills of Mortality. 5th ed. London: : Printed by John Martyn, Printer to the Royal Society, at the Sign of the Bell in St. Paul's Church-yard 1663.

4 Banerjee A, Chen S, Pasea L, et al. Excess deaths in people with cardiovascular diseases during the COVID-19 pandemic. Eur J Prev Cardiol Published Online First: 2021. doi:10.1093/eurjpc/zwaa155

5 Pitman RJ, Melegaro A, Gelb D, et al. Assessing the burden of influenza and other respiratory infections in England and Wales. J Infect 2007;54:530-8. doi:10.1016/j.jinf.2006.09.017

6 Ecclesiastes 9:11. In: The Bible: King James version.

7 Warren-Gash C, Smeeth L, Hayward AC. Influenza as a trigger for acute myocardial infarction or death from cardiovascular disease: a systematic review. Lancet Infect Dis 2009;9:601-10. doi:10.1016/S1473-3099(09)70233-6

8 NHS Coronary Heart Disease Overview. https://www.nhs.uk/conditions/coronary-heartdisease/ (accessed 9 Apr 2021).

9 Clar C, Oseni Z, Flowers N, et al. Influenza vaccines for preventing cardiovascular disease. Cochrane Database Syst Rev 2015;133:384. doi:10.1590/1516-3180.20151334T2

10 Collins R, Reith C, Emberson J, et al. Interpretation of the evidence for the efficacy and safety of statin therapy. Lancet 2016;388:2532-61. doi:10.1016/S0140-6736(16)31357-5

11 Collins R, Peto R, Hennekens C, et al. Aspirin in the primary and secondary prevention of vascular disease: collaborative meta-analysis of individual participant data from randomised trials. Lancet 2009;373:1849-60. doi:10.1016/S0140-6736(09)60503-1

12 Wynants L, Calster B Van, Bonten MM, et al. Systematic review and critical appraisal of prediction models for diagnosis and prognosis of COVID-19 infection. medRxiv 2020;:2020.03.24.20041020. doi:10.1101/2020.03.24.20041020

13 Taylor DA, Wallis K, Feki S, et al. Cardiovascular disease risk prediction in older people: a qualitative study. Br J Gen Pract 2021;:BJGP.2020.1038. doi:10.3399/BJGP.2020.1038

14 Hao Q, Tampi M, O’Donnell M, et al. Clopidogrel plus aspirin versus aspirin alone for acute minor ischaemic stroke or high risk transient ischaemic attack: Systematic review and metaanalysis. BMJ 2018;363. doi:10.1136/bmj.k5108

15 Horby PW, Pessoa-Amorim G, Staplin N, et al. Aspirin in patients admitted to hospital with COVID-19 (RECOVERY): a randomised, controlled, open-label, platform trial. medRxiv 2021;:2021.06.08.21258132. doi:10.1101/2021.06.08.21258132 